\title{
MULTI-SENSOR TRAFFIC DATA FUSION FOR CONGESTION DETECTION AND TRACKING
}

\author{
J. Gitahi ${ }^{1 *}$, M. Hahn ${ }^{1}$, M. Storz ${ }^{1}$, C. Bernhard ${ }^{2}$, M. Feldges ${ }^{3}$, R. Nordentoft ${ }^{4}$ \\ ${ }^{1}$ Faculty of Geomatics, Computer Science and Mathematics, Hochschule für Technik Stuttgart, Schellingstraße 24, 70174 \\ Stuttgart, Germany - (joseph.gitahi, michael.hahn, martin.storz) @ hft-stuttgart.de \\ ${ }^{2}$ ITS-United GmbH, Heinrich-Heine-Weg 21, D-73240 Wendlingen, Germany - christoph.bernhard@its-united.de \\ ${ }^{3}$ AVT-Consult GmbH, Am Tripser Wäldchen 9, D-52511 Geilenkirchen, Germany - michael.feldges@avt-consult.de \\ ${ }^{4}$ Traffic Network Solutions S.L., Comandant Benitez 2508028 Barcelona, Spain - rnordentoft@ trafficnow.eu
}

Commission I, WG I/6

KEYWORDS: Sensor and Data Fusion, FCD, Congestion Detection, Intelligent Transport Systems

\begin{abstract}
:
Traffic management applications including congestion detection and tracking rely on traffic from multiple sources to model the traffic conditions. The sources are either stationary sensors which include inductive loop detectors (ILD), radar stations and Bluetooth/WiFi/BLE sensors or Floating Car Data (FCD) from moving vehicles which transmit their locations and speeds. The different sources have their inherent strengths and weaknesses but when used together, they have the potential to provide traffic information with increased robustness. Multi-sensor data fusion has the potential to enhance the estimation of traffic state in real-time by reducing the uncertainty of individual sources, extending the temporal and spatial coverage and increasing the confidence of data inputs. In this study, we fuse data from different FCD providers to improve travel time and average segment speeds estimation. We use data from INRIX, HERE and TomTom FCD commercial services and fuse the speeds based on their confidence values and granularity on virtual sub-segments of $250 \mathrm{~m}$. Speeds differences between each pair of datasets are evaluated by calculating the absolute mean and standard deviation of differences. The evaluation of systematic differences is also performed for peak periods depending on the day of the week. INRIX FCD speeds are compared with ground truth spot speeds where both datasets are measured at a 1-minute interval which show good agreement with an error rate of between 8-20\%. Some issues that affect FCD accuracy which include data availability and reliability problems are identified and discussed.
\end{abstract}

\section{INTRODUCTION}

Road traffic monitoring relies on the accurate collection of information from different sources for comprehensive spatial and temporal traffic analysis. Speed, density and flow are three critical parameters in traffic analysis and should be measured or estimated accurately for use in traffic management systems. Traffic congestion is one of the major challenges facing transport systems. In this paper, we present the multi-sensor fusion of traffic data for end-of-congestion detection and tracking. Traffic data sources can be grouped into three categories. First, there are stationary radar sensors and inductive loop detectors (ILD) which measure multi-lane spot speeds, vehicle count, road occupancy, headway and in some cases the length of vehicles. In the second category, there is the Floating Car Data (FCD) transmitted by moving vehicles which provide travel times and average speeds on road sub-segments. The third category is data from Bluetooth/WiFi/BLE sensors mounted on consecutive locations on a road providing segment travel times and average speeds. While FCD and Bluetooth/WiFi/BLE sensors provide reliable travel times and average speeds, they are limited as they fail to capture multi-lane parameters and absolute volume of vehicles. On the other hand, radar and ILD sensors measure multi-lane absolute vehicle counts and spot speeds but do not provide accurate travel times and average speeds over road segments. In traffic management applications, multi-sensor data fusion techniques seek to use these different data sources to provide comprehensive and more reliable traffic state estimation. The different sources complement each other leading to increased accuracy and robustness. Data fusion also includes integrating similar datasets from different sources such as different FCD services to enhance the spatial and temporal coverage of traffic state estimation.

The work in this study is part of an ongoing project to detect and track end-of-congestion on a $14 \mathrm{~km}$ section of the A8 Highway (Autobahn A8) near Pforzheim, Baden-Württemberg in southwest Germany. In this stretch, traffic heading eastwards towards Stuttgart regularly experiences congestion due to a traffic bottleneck as the road narrows from three to two lanes. The location and planned installation of traffic monitoring sensors is shown in Figure 1.

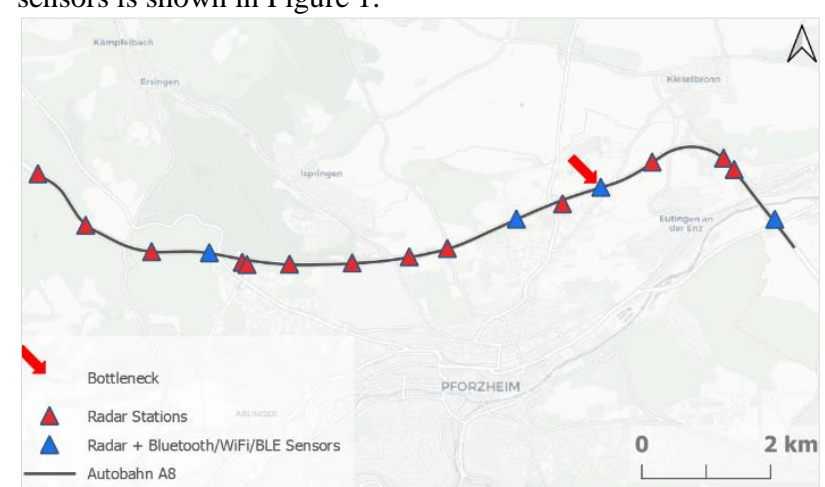

Figure 1: The planned installation of radar stations and Bluetooth/WiFi/BLE sensors on the A8 section. The bottleneck shows the point where the road narrows from three to two lanes.

\footnotetext{
* Corresponding author
} 
To enable enhanced detection and tracking of end-of-congestion, the road authorities plan to install radar stations and Bluetooth/WiFi/BLE sensors at short intervals along the highway section. The goal of this study is to use fusion techniques to integrate traffic data from the three data source categories for congestion modelling. In the first stage, we fuse data from three FCD services. The fusion techniques used in this step are also applicable to integrating Bluetooth/WiFi/BLE data with FCD as they both measure average speeds and travel times for road segments. The second step is fusing the reliable travel times and speeds from the first step with the multi-lane traffic counts obtained from radar stations. With the different traffic data sources fused, accurate estimates of speed, flow and density per lane for road segments essential for traffic modelling will be derived.

We show the necessary steps required to fuse FCD data to a common road segmentation network to estimate average speeds and travel times at high granularity. Since the Bluetooth/WiFi/BLE sensors and radar stations are not yet installed, a concept for fusion in the second stage is presented.

\section{BACKGROUND AND RELATED WORK}

The accuracy of travel times and speeds from FCD services depends on the number of probe vehicles sending timestamped geolocations and speeds. GPS and data transmission issues are other factors that affect the quality of traffic data. In cases where the penetration rate of probe vehicles is low or none, FCD services use historical data to predict traffic conditions which may not represent the actual traffic situations.

In highways with high penetration rates, FCD performs well in estimating travel times and shows better correlation with ground truth speeds from stationary detectors. In their study on expressways in Beijing, Zhao et al. (2009) observed a highly correlated relationship between remote traffic microwave sensors (RTMS) and FCD speeds on expressways with an R2 of 0.97 . Kondyli et al. (2018) compared the performance of various FCD from INRIX, HERE, STEWARD and BlueTOAD in estimating travel times on highways. The FCD speeds from all the providers successfully estimated travel times but with varying performance depending on the traffic conditions. In congested traffic HERE provided better estimates while the rest underestimated travel times. However, in less congested conditions, the other three providers had better estimates than HERE.

A critical issue to consider when using FCD for traffic state estimation is the latency in reporting real-time traffic conditions. Kim and Coifman (2014) found that INRIX FCD reported sudden drops in speeds 6 minutes later even though the confidence values included did not indicate lower quality speed data. Adu-Gyamfi et al. (2015) evaluated the reliability of FCD in detecting congestion on highways. While the FCD detected short-term congestion events with a 74\% accuracy, it lagged by 8 minutes compared to ground-truth measurements.

Multi-sensor data fusion techniques have been used in the scientific world by combining data from several sources to improve and provide reliable information. Traffic management applications employ these techniques for improved traffic state estimation using data from stationary detectors, FCD providers and Bluetooth/WiFi/BLE sensors. These data sources are combined with strengths in one source complementing the other's weaknesses to increase the robustness of the data. Fusing also enhances spatial and temporal coverage of traffic data. FCD and Bluetooth/WiFi/BLE sensors, for example, provide extensive coverage compared to stationary detectors which only provide information at point locations due to cost implications.
There are two major approaches to traffic data fusion. The first one is the data-model consistency approach which relies on measured data to calibrate models and in turn use the models to correct data so that it fits into models. Techniques like Kalman filters are used in this approach (Faouzi and Klein 2016; Wolfermann et al. 2011). Models combine data from stationary detectors and trajectories from moving sensors to determine density and speeds at specific locations. The major drawback of the modelling approach is that it requires making of several assumptions and there is a risk of running into overfitting problems during model calibration.

The second approach uses data-to-data consistency (Ou 2011) based on basic physical laws: speed $*$ time $=$ distance, density $*$ speed $=$ flow and the vehicle conservation law, Equation (1). In this approach traffic data from two sources is related based on the physical laws. One typical application is smoothing of noise of stationary detectors with noiseless FCD based travel time data.

$$
\rho(i, j)=\rho(i-1, j)+\frac{\Delta t}{L_{j}}(q(i, j-1)-q(i, j))
$$

Where: $\boldsymbol{\rho}=$ density, $\boldsymbol{q}=$ flow, $\boldsymbol{i}=1,2,3 \ldots$ represents discretetime, $\boldsymbol{j}$ indicates the location, $\boldsymbol{L}_{\boldsymbol{j}}$ is the length of the road segment $\boldsymbol{j}$ and $\Delta \boldsymbol{t}$ is the span of one discrete-time.

Similar categories of sensors are also fused to improve the accuracy of traffic state estimation. Use cases include fusion for better estimation of travel times from different FCD providers. This is achieved by calculating the weighted mean of several travel-times estimators where the weights are a function of variance or covariance of estimators, or a function of the data source reliability (Faouzi and Klein 2016). There are many fusion techniques available but the choice of fusion strategy to use depends on data sources availability, ease of computation, traffic management application and the desired results which may vary depending on the traffic management application. In this study, the time of computation is essential as the end-of-congestion detection is required at a high temporal resolution of one minute.

\section{DATA AND METHODOLOGY}

\subsection{Data}

On the A8 highway section, three FCD services are available which are INRIX, TomTom and HERE each reporting traffic data at a one-minute interval. The data, retrieved from each of their respective traffic flow APIs, includes average segment speeds, travel times, free-flow speeds and confidence values as a measure of confidence for real-time data. Additionally, INRIX has a score indicating whether the traffic data is real-time, historical or a blend of both and also reports average speeds based on historical data at a particular time-of-day and day-of-week.

INRIX data is based on the standard Traffic Messaging Channel (TMC) segments and INRIX eXtreme Definition segments (XDS) which have higher granularity levels. HERE traffic data is based on TMC segments while TomTom data can be accessed using TMC, Open Location Referencing (OpenLr) or manually defined points on desired road segments. In each case the road segmenting scheme with the highest resolution was chosen as follows, INRIX XDS segments ranging from 140m - 1600m, HERE TMC segments ranging from $200 \mathrm{~m}-12000 \mathrm{~m}$ and TomTom segments ranging from $280 \mathrm{~m}-2700 \mathrm{~m}$ defined by manually placing points on the road section. The segments from each FCD service are stored in the database as spatial tables and 
are shown in Figure 2. Due to the project's limited access to HERE and TomTom services, contemporaneous speeds from the three services are retrieved at seven-minute intervals. A twoweek dataset running from $18^{\text {th }}$ February to $3^{\text {rd }}$ March 2020 is used in the study.

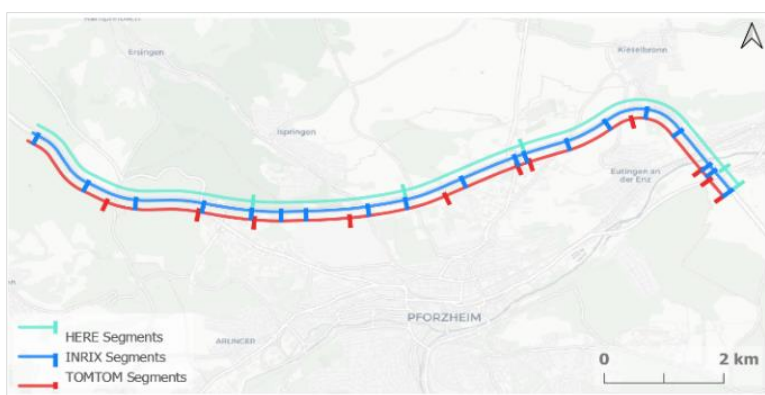

Figure 2: HERE, INRIX and TomTom road segments on the A8 highway. The line geometries have been offset for easier visualization and comparison of their varying lengths.

There are stationary traffic cross-sectional measurement stations operated by the state road department, Landesstelle für Straßentechnik, Baden-Württemberg. The stations provide spot speeds and vehicle flow at one-minute intervals for three categories of vehicles; cars, lorries and others. There are 7 stations on the study location, 3 on the highway and 4 on the onramps and off-ramps as shown in Figure 3. They provide speed and flow measurements at a one-minute interval. This dataset is used as ground truth to evaluate another set of INRIX speeds retrieved simultaneously at a one-minute interval on April 2020.

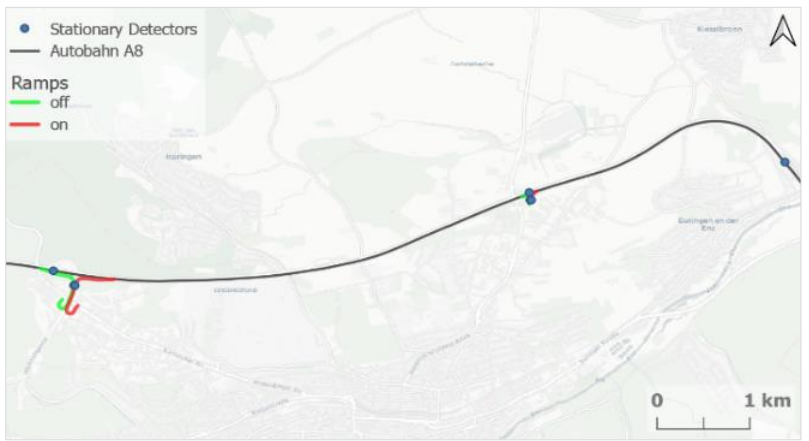

Figure 3: Stationary cross-sectional measurement stations.

A second dataset from Ajaccio, the capital city of Corsica, is used in the study. This consists of data from radar sensors, Bluetooth sensors and TomTom. HERE FCD does not cover this location and we do not have access to INRIX. Bluetooth sensors placed at upstream and downstream boundaries of road sub-segments provide average speeds, travel times and the number of probe vehicles used to measure these parameters. This is comparable to the FCD dataset and is used as ground truth to evaluate the accuracy of TomTom traffic flow data Figure 4. This urban dataset is retrieved at two-minute intervals and the comparative studies are performed for data collected in March 2020.

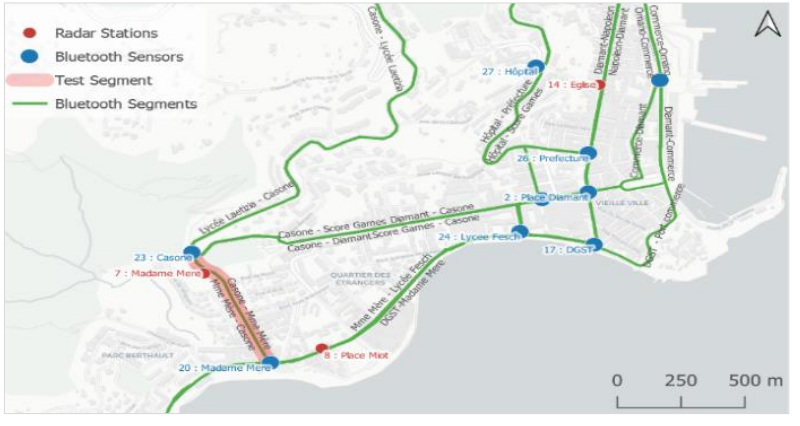

Figure 4: Radar stations and Bluetooth sensors placed in Ajaccio's road network. The segment highlighted in red will be used to compare TomTom FCD speeds against the Bluetooth sensors speeds in both directions.

\subsection{Methodology}

\subsubsection{Data Retrieval and Storage}

All the datasets used in the project are managed using a PostgreSQL database management system (DBMS) extended with PostGIS for spatial data support. The datasets consist of the static road segments and dynamic traffic tables for each of the FCD service updated periodically. Datasets from the three FCD providers, the stationary detectors and the Bluetooth sensors are retrieved from their respective APIs in JSON and DATEX formats. Python and NodeJS scripts are used to fetch, pre-process and store the data.

\subsubsection{Geometry Preparation}

Each of the road segments from the FCD services on the A8 section vary in length, start and end nodes. To fuse these datasets, the road section is segmented to $250 \mathrm{~m}$ virtual sub-segments using the bottleneck location as a point of reference and OpenStreetMap road geometry. The segment length and the boundaries are determined by the project requirements to monitor congestion build-up upstream from the bottleneck location with high granularity. In total 53 segments are created, with segments S1-S38 from the start of the highway section to the bottleneck and segments S39-S53 after the bottleneck to the end. Some of the subsegments are shown in Figure 5.

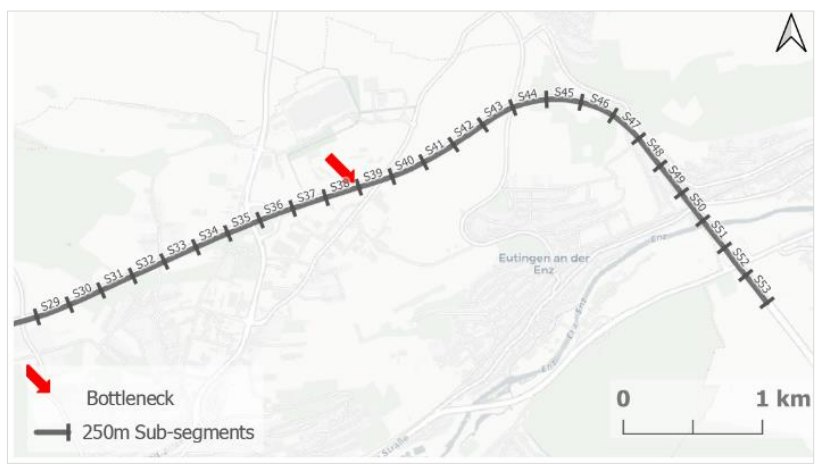

Figure 5: Subdividing the A8 highway section into virtual $250 \mathrm{~m}$ sub-segments.

\subsubsection{FCD Data Fusion}

In this step, the dynamic traffic datasets from each of the services are related to their respective segment geometry through a SQL join using a segment id. This enables spatial operations necessary for the next step which assigns traffic parameters from each service to the $250 \mathrm{~m}$ sub-segments for each timestep. In this spatial-temporal fusion, a calculation of the weighted average speeds per $250 \mathrm{~m}$ sub-segment is carried out using the confidence 
values and length of the source segment as weights. The weight parameters are a function of data source reliability as reported by FCD service providers and the granularity of their corresponding segments. This is calculated as shown in Equation (2):

$$
\bar{x}=\frac{\sum_{i=1}^{n}\left(v_{i} c_{i} \frac{1}{i_{i}}\right)}{\sum_{i=1}^{n}\left(c_{i} \frac{1}{L_{i}}\right)}
$$

where: $\overline{\boldsymbol{x}}=$ weighted average speed, $\boldsymbol{v}=$ speed, $\boldsymbol{c}=$ confidence, $\boldsymbol{L}=$ Length of segment

3.2.4 Concept for FCD, Bluetooth/WiFi/BLE and Radar Sensors Fusion

The planned installation of Radar and Bluetooth/WiFi/BLE sensors on the A8 section will enhance the spatial coverage of traffic monitoring. Placement of the sensors at short segments as shown in Figure 1 is expected to increase the accuracy of traffic state estimation. Radar stations will provide spot speeds and vehicle counts per lane while Bluetooth/WiFi/BLE will provide average speeds and travel times on segments. The end goal is to fuse data from these sensors and FCD to obtain reliable multilane speeds, densities and flows for congestion modelling.

For stationary detectors placed consecutively along a road section at short segments of between $1-1.5 \mathrm{~km}$, the segment speeds and flows can be estimated by calculating the average of speeds and flows from detectors placed at the start and end of a segment. Since the segments between consecutive detectors on the A8 section are short, with lengths measuring approximately $1 \mathrm{~km}$, the average speeds $(\mathrm{km} / \mathrm{h})$, flows (vehicles $/ \mathrm{h}$ ) and densities (vehicles $/ \mathrm{km}$ ) for each lane can be estimated using Equations (3), (4) and (5) respectively as proposed by Qiu et al. (2009):

$$
\begin{gathered}
v\left(x_{i,} x_{i+1}, k\right)=\frac{1}{2}\left(v\left(x_{i}, k\right)+v\left(x_{i+1}, k\right)\right) \\
q\left(x_{i,} x_{i+1}, k\right)=\frac{1}{2}\left(q\left(x_{i}, k\right)+q\left(x_{i+1}, k\right)\right) \\
\rho\left(x_{i,} x_{i+1}, k\right)=\frac{q\left(x_{i,} x_{i+1}, k\right)}{v\left(x_{i}, x_{i+1}, k\right)}
\end{gathered}
$$

where:

$i$ is detector station index

$v(x, k)$ is spot-based speed at location $x$ during time interval $k$

$q(x, k)$ is spot-based flow at location $x$ during time interval $k$ $v\left(x_{i}, x_{i+1}, k\right)$ is segment-based speed at the section between $x_{i}$ and $x_{i+1}$, during time interval $k$

$q\left(x_{i}, x_{i+1}, k\right)$ is segment-based flow at the section between $x_{i}$ and $x_{i+1}$, during time interval $k$

$\rho\left(x_{i}, x_{i+1}, k\right)$ is segment-based density at the section between $x_{i}$ and $x_{i+1}$ during time interval $k$

The assumption while using this technique is that there exists no on and off-ramps in between the detector stations. As some of the segments on the section of the highway have ramps, the vehicle count changes will have to be factored in for accurate density calculation.

The second step will be to fuse travel times from FCD and Bluetooth/WiFi/BLE sensors with speeds measured by radar stations for improving flow and density estimations in the first step for each road segment. Data to data consistency models such as those proposed by $\mathrm{Ou}$ (2011) will be evaluated based on their results and computational time.

\subsubsection{Evaluation Methodology}

As discussed in Section 2, FCD quality depends on the number of vehicles transmitting their locations and speeds. Historical data is used to predict traffic conditions where real-time information is not present or to supplement real-time data. For HERE FCD, the confidence values are 0.7-1.0 for real-time speeds, $0.5-0.7$ for historical speeds, less than 0.5 indicates the speed limit and a value of -1.0 indicates a closed road segment. INRIX FCD uses score values 30,20 and 10 to indicate whether the speeds are real-time, a blend of real-time and historical or historical respectively. We use the FCD speeds and the weighted speed averages for the $250 \mathrm{~m}$ virtual sub-segments to identify peak and off-peak periods at points of interest for both weekdays and weekends. Due to the high volume of traffic during peak periods, it is expected that the penetration rate of probe vehicles is high for each service and that the reported speeds have high confidence values. Identification of the peak periods is also required to evaluate the performance of FCD in detecting and tracking traffic congestion. The identification is done through a visual analysis of plotted time-of-day average speeds over time for segments of interest. The evaluations are thus performed for the entire study periods and peak periods for both study locations. Speed differences from the three FCD services and the weighted average speeds on all segments along the road section are plotted for visual comparison for both peak and off-peak periods. This is used to identify hours of the day when the FCD services detect speed drops and congestion build-up.

To evaluate systematic differences between speeds from the three FCD services, absolute mean and standard deviation of speed differences are calculated for each pair of FCD datasets. The absolute mean difference is calculated as the sum of absolute speed differences over time divided by the number of observations as used by Chase et al. (2012) and Anuar et al. (2015) to compare FCD speeds from different sources. The equations for calculating the absolute mean and standard deviation of speeds differences are shown in Equations (6) and (7) respectively.

$$
\bar{d}=\frac{1}{n} \sum_{i=1}^{n}\left|d_{i}\right|
$$

$$
\sigma=\sqrt{\frac{\sum_{i=1}^{n}\left(\left|d_{i}\right|-\bar{d}\right)^{2}}{n-1}}
$$

Where: $n$ is the number of samples, $\left|d_{i}\right|=\left|x_{i}-y_{i}\right|, x_{i}$ and $y_{i}$ are different FCD speeds

TomTom FCD is evaluated against ground truth segment speeds from Bluetooth sensors in Ajaccio while on the A8, INRIX FCD is evaluated against speeds measured by stationary detectors on the A8. We calculate mean absolute percentage error (MAPE) and root mean square error (RMSE) as used by $\mathrm{Hu}$ et al. (2016) and Anuar et al. (2015) to evaluate FCD speed deviations from ground truth measurements. MAPE and RMSE calculations are shown in Equations (8) and (9) respectively. 


$$
\begin{gathered}
M A P E=\frac{1}{n} \sum_{i=1}^{n}\left|\frac{V_{G T}-V_{F C D}}{V_{F C D}}\right| \times 100 \\
R M S E=\sqrt{\frac{1}{n} \sum_{i=1}^{n}\left(V_{G T}-V_{F C D}\right)^{2}}
\end{gathered}
$$

Where: $n$ is the number of samples, $V_{G T}$ the ground truth speeds and $V_{F C D}$ the FCD speeds.

\section{RESULTS ANALYSIS}

On our main study location, the A8 highway section, the $250 \mathrm{~m}$ sub-segment before the bottleneck, sub-segment " $S 38$ ", is of interest due to regular traffic congestion. We calculate time-ofday average speeds for each service to identify peak and off-peak periods. From Figure 6, the major peak period on weekdays is identified to start from $06: 00 \mathrm{hrs}$ to $13: 00 \mathrm{hrs}$ which is the period that most cars drive towards Stuttgart city. During the weekends, the major peak starts from $07: 00 \mathrm{hrs}$ to $11: 00 \mathrm{hrs}$ as shown in Figure 7. On this section, speeds of less than $40 \mathrm{~km} / \mathrm{h}$ indicate congestion. Peak periods are characterized by higher penetration rates of probe vehicles and thus higher quality data is expected. We only consider the major peak periods.

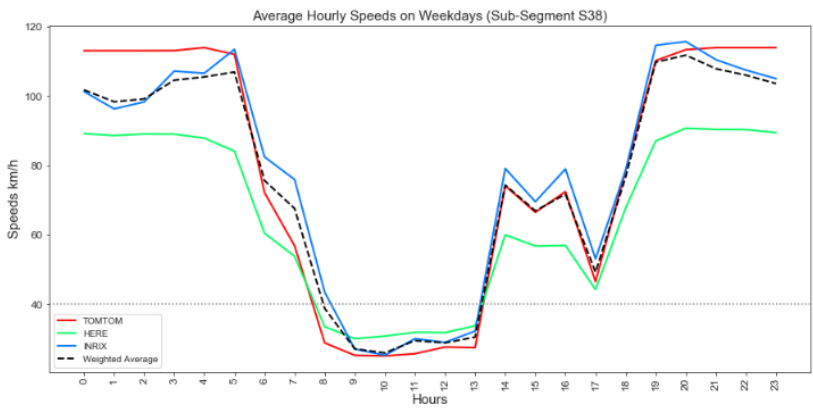

Figure 6: Time-of-day average speeds on weekdays for the virtual sub-segment before the bottleneck location (S38).

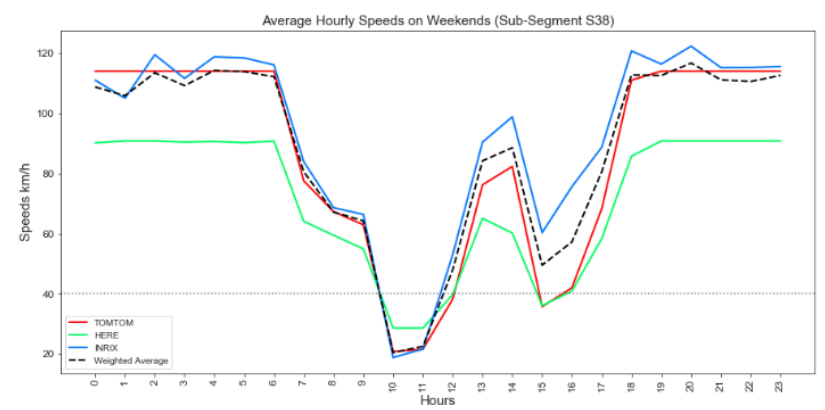

Figure 7: Time-of-day average speeds on weekends for the virtual sub-segment before the bottleneck location (S38).

We compare the reported confidence values over the study period for this segment. In Figure 8 HERE consistently reports very high values throughout while INRIX shows the highest variance of confidence which is consistent with time-of-day average speeds. TomTom values vary but by a small margin during the daytime. This trend is similar for the entire road section. From INRIX scores, $99 \%$ of the speeds reported on both weekdays and weekends for all segments were real-time.

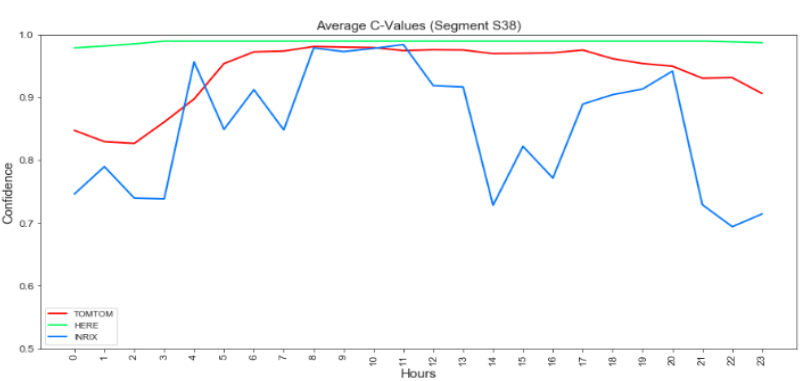

Figure 8: Time-of-day hourly average confidence values reported by the FCD services on Segment S38 during weekdays.

Speed variations between pairs of FCD services are compared by calculating the absolute mean and standard deviations of speed differences. The calculations are done separately for weekends and weekdays and their respective peak periods, as shown in Table 1. As expected, the high volume of vehicles during the peak periods improve the probe rate for all the FCD services which results in reduced variations in speed differences. The influence of spatial resolution FCD is also seen when the speeds are fused to a common segment. At sub-segment S38, HERE FCD has the lowest spatial resolution with longer segments among the three services and hence the higher variations in speeds when compared to the rest. On this sub-segment, the lengths of corresponding HERE, TomTom and INRIX segments are $3900 \mathrm{~m}, 2720 \mathrm{~m}$ and $750 \mathrm{~m}$ respectively and are compared in Figure 9.

Table 1: Absolute mean and standard deviations of FCD speeds over different periods for sub-segment S38

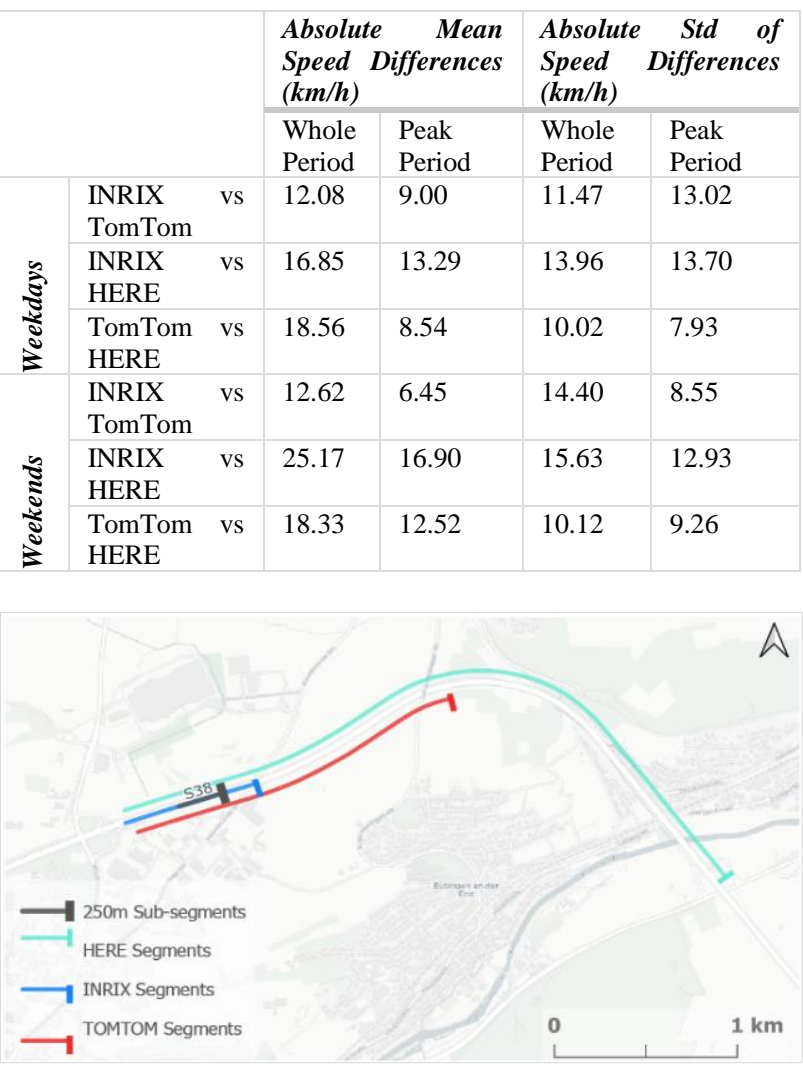

Figure 9: A length comparison of INRIX, TomTom and HERE segments with the virtual sub-segment S38. 
The results for absolute mean and standard deviations calculations for the entire A8 highway section are shown in Table 2. Generally, the speeds show less variation from each other during peak hours apart from INRIX and HERE pairs where the differences increase. From the time-of-day average speeds plots in Figure 10 and Figure 11, INRIX and HERE speeds show better agreement during off-peak periods while TomTom reports higher speeds. However, as speeds decrease during peak hours, HERE reports lower speeds than the rest. This could be as a result of the longer TMC segments generalizing HERE speeds more than INRIX and TomTom which have higher granularities. The weighted average speeds are highly correlated with INRIX speeds as it has the highest granularity among the three FCD services. The correlation is slightly lower at night which corresponds to the lower confidence levels reported by INRIX.

Table 2:Absolute mean and standard deviations of FCD speeds over different periods for the entire road section

\begin{tabular}{|c|c|c|c|c|c|c|}
\hline & \multicolumn{2}{|c|}{$\begin{array}{l}\text { Absolute Mean } \\
\text { Speed Differences } \\
(\mathrm{km} / \mathrm{h})\end{array}$} & \multicolumn{2}{|c|}{$\begin{array}{l}\text { Absolute } \quad \text { Std of } \\
\begin{array}{l}\text { Speed } \\
(\mathrm{km} / \mathrm{h})\end{array}\end{array}$} \\
\hline & & & $\begin{array}{l}\text { Whole } \\
\text { Period }\end{array}$ & $\begin{array}{l}\text { Peak } \\
\text { Period }\end{array}$ & $\begin{array}{l}\text { Whole } \\
\text { Period }\end{array}$ & $\begin{array}{l}\text { Peak } \\
\text { Period }\end{array}$ \\
\hline \multirow{3}{*}{ 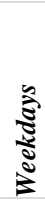 } & $\begin{array}{l}\text { INRIX } \\
\text { TomTom }\end{array}$ & vs & 14.33 & 10.63 & 13.07 & 12.52 \\
\hline & $\begin{array}{l}\text { INRIX } \\
\text { HERE }\end{array}$ & vs & 12.29 & 13.43 & 12.45 & 14.96 \\
\hline & $\begin{array}{l}\text { TomTom } \\
\text { HERE }\end{array}$ & vs & 13.82 & 12.98 & 13.43 & 15.51 \\
\hline \multirow{3}{*}{ 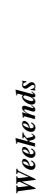 } & $\begin{array}{l}\text { INRIX } \\
\text { TomTom }\end{array}$ & vs & 9.80 & 8.26 & 9.49 & 8.71 \\
\hline & $\begin{array}{l}\text { INRIX } \\
\text { HERE }\end{array}$ & vs & 10.71 & 10.82 & 10.58 & 12.23 \\
\hline & $\begin{array}{l}\text { TomTom } \\
\text { HERE }\end{array}$ & vs & 8.35 & 9.83 & 11.23 & 14.67 \\
\hline
\end{tabular}

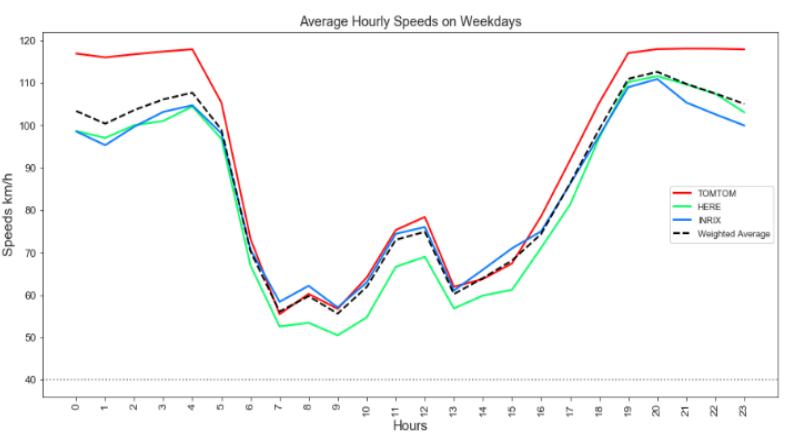

Figure 10: Time-of-day average speeds on weekdays for the entire road section.

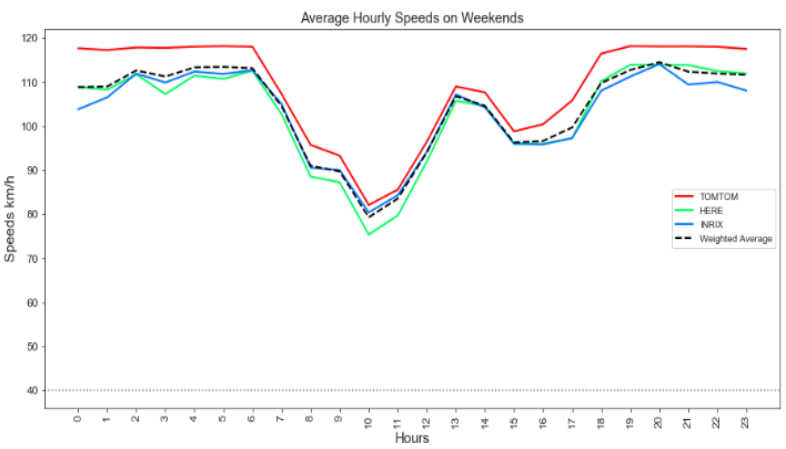

Figure 11: Time-of-day average speeds on weekends for the entire road section
The performance of FCD is further evaluated by comparing the space-mean speeds with ground truth time-mean speeds. On the A8 we compare INRIX FCD which is available at both high spatial and temporal resolutions with ground measurements from the stationary detectors. In this ground truth dataset, speeds from the three categories of vehicles are averaged for comparison. In the MAPE and RMSE results shown in

Table 3, there is no significant variance between the whole period and peak period. The last segment, which is the only one not on an intersection shows the best agreement where FCD speeds are only $8.4 \%$ different than ground truth speeds during the peak period. The length of the segment does not have an impact on the performance of the FCD speeds but flow interruptions on intersections yield higher errors.

Table 3: A comparison of INRIX FCD performance compared to ground truth speeds on different segments. The segments apart from the last one in the table lie between the OFF and ON ramps on intersections.

\begin{tabular}{|l|l|l|l|l|l|l|}
\hline \multicolumn{2}{|c|}{} & \multicolumn{2}{l|}{$\begin{array}{l}\text { Weekdays } \\
\text { (Whole Period) }\end{array}$} & \multicolumn{2}{l|}{ Weekdays Peak } \\
\hline $\begin{array}{l}\text { INRIX } \\
\text { Segments }\end{array}$ & Ramp & $\begin{array}{l}\text { Length } \\
(\mathrm{m})\end{array}$ & $\begin{array}{l}\text { MAPE } \\
(\%)\end{array}$ & $\begin{array}{l}\text { RMSE } \\
(\mathrm{km} / \mathrm{h})\end{array}$ & $\begin{array}{l}\text { MAPE } \\
(\%)\end{array}$ & $\begin{array}{l}\text { RMSE } \\
(\mathrm{km} / \mathrm{h})\end{array}$ \\
\hline $\mathbf{3 6 5 6 1 7 8 1 1}$ & OFF & 289 & 20.64 & 14.20 & 20.68 & 14.62 \\
\hline $\mathbf{3 6 5 6 5 6 3 2 5}$ & & 168 & 13.26 & 17.13 & 12.46 & 16.61 \\
\hline $\mathbf{3 6 5 6 4 6 6 0 6}$ & ON & 166 & 18.77 & 16.96 & 18.08 & 15.86 \\
\hline $\mathbf{3 6 5 7 3 7 4 4 8}$ & OFF & 459 & 15.37 & 13.01 & 14.16 & 12.19 \\
\hline $\mathbf{3 6 5 6 3 3 0 5 4}$ & & 810 & 11.00 & 15.03 & 10.98 & 15.21 \\
\hline $\mathbf{3 6 5 7 3 6 3 8 8}$ & ON & 483 & 13.25 & 12.61 & 12.43 & 11.61 \\
\hline $\mathbf{3 6 5 7 5 7 8 5 9}$ & & 775 & $\mathbf{8 . 6 8}$ & $\mathbf{1 0 . 5 0}$ & $\mathbf{8 . 4}$ & $\mathbf{9 . 6 2}$ \\
\hline
\end{tabular}

In all segments, INRIX reports higher speeds than the ground truth speeds averaged for the three categories of vehicles. When compared with individual vehicle categories in Figure 12, INRIX speeds are closest to speeds reported for the car category indicating that a large proportion of probe vehicles in this section are passenger cars.

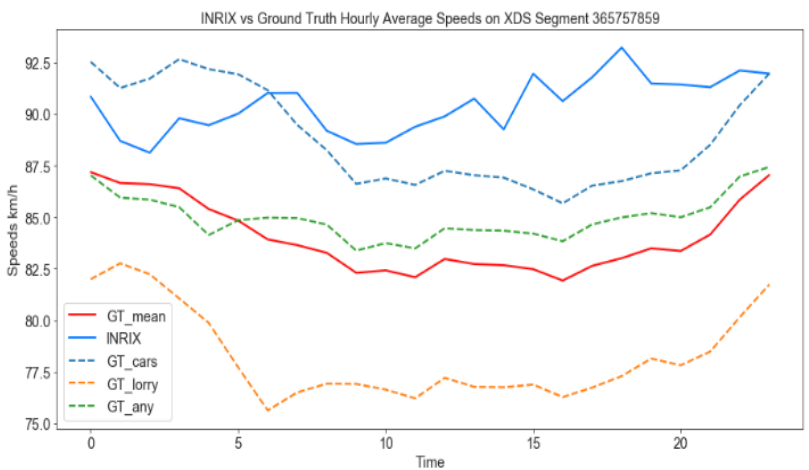

Figure 12: Comparison of time-of-day average speeds from INRIX FCD stationary detectors on INRIX segment XDS 365757859 (775m). The individual vehicle category speeds from the detectors are shown in dashed lines.

In Ajaccio, we compare speed TomTom deviations from the segment speeds measured by Bluetooth sensors. The comparison is on a $477 \mathrm{~m}$ segment near the city centre for both driving directions. From the calculated time-of-day speed averages, TomTom on both weekdays and weekends underestimates the speeds especially during the morning and evening peak periods on weekdays as shown in Figure 13 and Figure 14. This trend is observed on both driving directions consistently which could 
indicate TomTom's lower penetration rate. The confidence values for both sets of speeds have a similar trend rising in the morning and dipping after evening hour traffic but the ground truth speeds from the Bluetooth sensors report lower values. Based on these confidence values and the error rate which is between $28-30 \%$ in Table 4, the quality of both datasets to estimate real-time conditions would be difficult to ascertain unless further quality tests are conducted.

Table 4: A comparison of error rates on space-mean speeds from TomTom FCD compared to Bluetooth speeds on both driving Casone - Madame Mère towards the city and Madame Mère - Casone out of the city.

\begin{tabular}{|l|l|l|l|l|}
\hline & \multicolumn{2}{|l|}{ Weekdays } & \multicolumn{2}{l|}{ Weekends } \\
\hline Direction & $\begin{array}{l}\text { MAPE } \\
(\%)\end{array}$ & $\begin{array}{l}\text { RMSE } \\
(\mathrm{km} / \mathrm{h})\end{array}$ & $\begin{array}{l}\text { MAPE } \\
(\%)\end{array}$ & $\begin{array}{l}\text { RMSE } \\
(\mathrm{km} / \mathrm{h})\end{array}$ \\
\hline Casone - Madame Mère & 35.11 & 10.36 & 28.29 & 16.02 \\
\hline Madame Mère - Casone & 28.58 & 11.45 & 29.67 & 12.94 \\
\hline
\end{tabular}

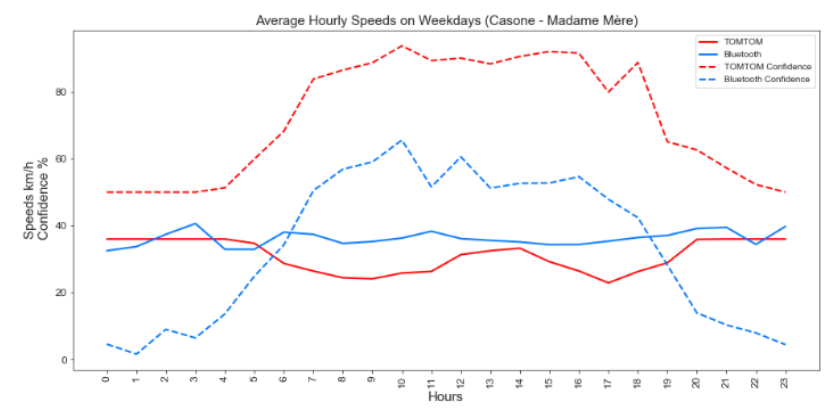

Figure 13: A comparison of TomTom and Bluetooth time-ofday average speeds and the confidence values for traffic driving towards the city centre.

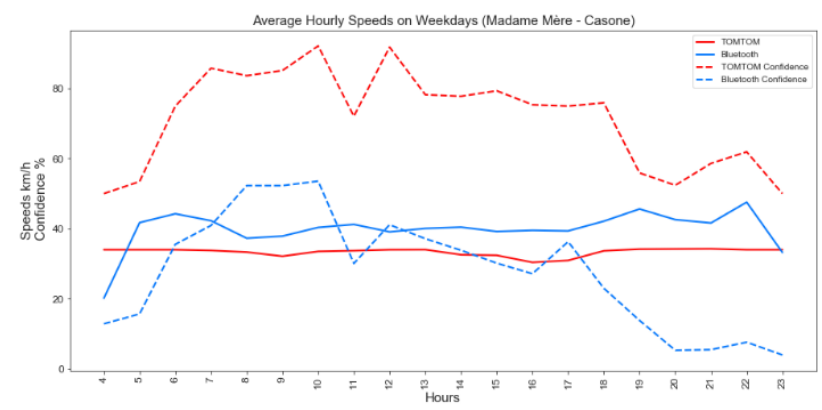

Figure 14: A comparison of TomTom and Bluetooth time-ofday average speeds and confidence values for traffic driving away from the city centre.

\section{CONCLUSIONS}

FCD is an important source of space-mean speeds and travel times, at higher spatial coverage and lesser cost than physically installed detectors such as radar. However, they are sometimes affected by reliability issues. The confidence values reported by the commercial services are hard to validate unless the speeds are compared with ground truth speeds. For example, on date $24^{\text {th }}$ February 2020, when a section of the A8 highway was closed for construction, HERE was still reporting speeds with a confidence value of 0.71. The MAPE and RMSE scores of INRIX speeds on the main section of the highway against the ground truth speeds, suggest that FCD has the potential to estimate speeds reliably and could be used for real-time congestion-detection and tracking. However, the performance decreases for road segments on intersections and FCD should not be used as the only source of speeds.

Long term evaluations of speeds from FCD services against highquality ground truth data is needed to determine their accuracy and completeness before using the data in traffic management applications. Acceptable error rates for each traffic use case should also be defined and used as a threshold to filter out lowquality data. The FCD performance also depends on the location and time of the day and day of the week and consequently there is a need to perform evaluations at the highest possible spatial resolution.

Multi-sensor fusion techniques have the potential of increasing the robustness of travel times and mean segment speeds estimation where one source complements lower quality data from another. The temporal coverage is also enhanced as data from more probe vehicles is used. The choice of fusion algorithms for combining data from different sources should be guided by the traffic management application. Processing time and the computational resources needed should be considered especially where real-time traffic estimation is required. Extensive validation and quality control of the data sources is essential as this has a significant impact on the accuracy of the fused data.

\section{ACKNOWLEDGEMENT}

The authors would like to thank the Ministry of Transport BadenWürttemberg for supporting the project End-of-congestion alarm (Stauende-Alarm) within the funding line MobiArch BW Mobility data architecture for innovative applications (Mobilitätsdatenarchitektur für innovative Anwendungen).

\section{REFERENCES}

Adu-Gyamfi, Yaw; Sharma, Anuj; Knickerbocker, Skylar; Hawkins, Neal; Jackson, Michael (2015): Reliability of Probe Speed Data for Detecting Congestion Trends. In : 2015 IEEE 18th International Conference on Intelligent Transportation Systems (ITSC). 15 - 18 Sept. 2015, Las Palmas, Gran Canaria, Spain, pp. 2243-2249.

Anuar, Khairul; Habtemichael, Filmon; Cetin, Mecit (2015): Estimating Traffic Flow Rate on Freeways from Probe Vehicle Data and Fundamental Diagram. In : 2015 IEEE 18th International Conference on Intelligent Transportation Systems (ITSC). 15 - 18 Sept. 2015, Las Palmas, Gran Canaria, Spain. pp. 2921-2926.

Chase, R. Thomas; Williams, Billy M.; Rouphail, Nagui M.; Kim, SangKey (2012): Comparative Evaluation of Reported Speeds from Corresponding Fixed-Point and Probe-Based Detection Systems. In Transportation Research Record 2308 (1), pp. 110-119. DOI: 10.3141/2308-12.

Faouzi, Nour-Eddin El; Klein, Lawrence A. (2016): Data Fusion for ITS. Techniques and Research Needs. In Transportation Research Procedia 15, pp. 495-512. DOI: 10.1016/j.trpro.2016.06.042.

Hu, Jia; Fontaine, Michael D.; Ma, Jiaqi (2016): Quality of Private Sector Travel-Time Data on Arterials. In J. Transp. Eng. 142 (4), p. 4016010. DOI: 10.1061/(ASCE)TE.19435436.0000815 . 
Kim, Seoungbum; Coifman, Benjamin (2014): Comparing INRIX speed data against concurrent loop detector stations over several months. In Transportation Research Part C: Emerging Technologies 49, pp. 59-72. DOI: 10.1016/j.trc.2014.10.002.

Kondyli, Alexandra; St. George, Bryan; Elefteriadou, Lily (2018): Comparison of travel time measurement methods along freeway and arterial facilities. In Transportation Letters 10 (4), pp. 215-228. DOI: $10.1080 / 19427867.2016 .1245259$.

Ou, Qing (2011): Fusing heterogeneous traffic data. Parsimonious approaches using data-data consistency. Delft: TRAIL Research School (Trail Thesis Series, T2011/5, 165 p.).

Qiu, Tony Z.; Lu, Xiao-Yun; Chow, Andy H.F. (2009): Realtime Density Estimation on Freeways With Loop Detector and Probe Data. In IFAC Proceedings Volumes 42 (15), pp. 298-303. DOI: 10.3182/20090902-3-US-2007.0051.

Wolfermann, Axel; Mehran, Babak; Kuwahara, Masao (2011) Data fusion for traffic flow estimation at intersections. In : 2nd International Workshop on Traffic Data Collection \& its Standardisation. Available online at https://elib.dlr.de/74195/.

Zhao, Nale; Yu, Lei; Zhao, Hui; Guo, Jifu; Wen, Huimin (2009): Analysis of Traffic Flow Characteristics on Ring Road Expressways in Beijing. In Transportation Research Record 2124 (1), pp. 178-185. DOI: 10.3141/2124-17. 